LBL 33232

PUPT 1355

November, 1992

\title{
SOME NEW/OLD APPROACHES TO QCD
}

\author{
DAVID J. GROSS ${ }^{\dagger}$
}

$L B L$

\begin{abstract}
I discuss some recent attempts to revive two old ideas regarding an analytic approach to QCD-the development of a string representation of the theory and the large $N$ limit of QCD.
\end{abstract}

* Talk delivered at the Meeting on Integrable Quantum Field Theories, Villa Olmo and at STRINGS 1992, Rome, September 1992. This work was supported in part by the Director, Office of Energy Research, Office of High Energy and Nuclear Physics, Division of High Energy Physics of the U.S. Department of Energy under Contract DE-AC03-76SF00098 and in part by the National Science Foundation under grant PHY90-21984.

$\dagger$ On leave from Princeton University, Princeton, New Jersey. 


\section{Disclaimer}

This document was prepared as an account of work sponsored by the United States Government. Neither the United States Government nor any agency thereof, nor The Regents of the University of California, nor any of their employees, makes any warranty, express or implied, or assumes any legal liability or responsibility for the accuracy, completeness, or usefulness of any information, apparatus, product, or process disclosed, or represents that its use would not infringe privately owned rights. Reference herein to any specific commercial products process, or service by its trade name, trademark, manufacturer, or otherwise, does not necessarily constitute or imply its endorsement, recommendation, or favoring by the United States Government or any agency thereof, or The Regents of the University of California. The views and opinions of authors expressed herein do not necessarily state or reflect those of the United States Government or any agency thereof of The Regents of the University of California and shall not be used for advertising or product endorsement purposes.

Lawrence Berkeley Laboratory is an equal opportunity employer. 


\section{Introduction}

In this lecture I shall discuss some recent attempts to revive some old ideas to address the problem of solving QCD. I believe that it is timely to return to this problem which has been woefully neglected for the last decade. QCD is a permanent part of the theoretical landscape and eventually we will have to develop analytic tools for dealing with the theory in the infra-red. Lattice techniques are useful but they have not yet lived up to their promise. Even if one manages to derive the hadronic spectrum numerically, to an accuracy of $10 \%$ or even $1 \%$, we will not be truly satisfied unless we have some analytic understanding of the results. Also, lattice Monte-Carlo methods can only be used to answer a small set of questions. Many issues of great conceptual and practical interest-in particular the calculation of scattering amplitudes, are thus far beyond lattice control. Any progress in controlling QCD in an explicit analytic fashion would be of great conceptual value. It would also be of great practical aid to experimentalists, who must use rather ad-hoc and primitive models of QCD scattering amplitudes to estimate the backgrounds to interesting new physics.

I will discuss an attempt to derive a string representation of QCD and a revival of the large $N$ approach to QCD. Both of these ideas have a long history, many theorist-years have been devoted to their pursuitso far with little success. I believe that it is time to try again. In part this is because of the progress in the last few years in string theory. Our increased understanding of string theory should make the attempt to discover a stringy representation of QCD easier, and the methods explored in matrix models might be employed to study the large $\mathrm{N}$ limit of QCD. For both political and intellectual reasons I fervently urge string theorists to try their hand at these tasks. 


\section{QCD as a String Theory}

It is an old idea that QCD might be represented as a string theory. This notion dates back even before the development of QCD. Indeed, string theory itself was stumbled on in an attempt to guess simple mathematical representations of strong interaction scattering amplitudes which embodied some of the features gleamed from the experiments of the 1960's. Many of the properties of hadrons are understandable if we picture the hadrons as string-like flux tubes. This picture is consistent with linear confinement, with the remarkably linear Regge trajectories and with the approximate duality of hadronic scattering amplitudes.

Within QCD itself there is internal, theoretical support for this idea. First, the $\frac{1}{N}$ expansion of weak coupling perturbation theory can be interpreted as corresponding to an expansion of an equivalent string theory in which the string coupling is given by $\frac{1}{N}$. This is the famous result of 't-Hooft's analysis of the $\frac{1}{N}$ expansion of perturbative QCD [1]. The same is true for any matrix model-i.e. a model invariant under $S U(N)$ or $U(N)$, in which the basic dynamical variable is a matrix in the adjoint representation of the group. The Feynman graphs in such a theory can be represented as triangulations of a two dimensional surface. This is achieved by writing the gluon propagator as a double index line and tiling the graph with plaquettes that cover the closed index loops. 't-Hooft's principal result was that one can use $\frac{1}{N}$ to pick out the topology, i.e. the genus=number of handles, of the surface, since a diagram which corresponds to a genus $G$ Riemann surface is weighted by $\left(\frac{1}{N}\right)^{2 G-2}$. The leading order in the expansion of the free energy in powers of $\frac{1}{N}$ is proportional to $N^{2}$ (reasonable since there are $N^{2}$ gluons, and is given by the planar graphs of the theory.

Another bit of evidence comes from the strong coupling lattice formulation of the theory. The strong coupling expansion of the free energy can indeed be represented as a sum over surfaces [5]. Again there is a natural large $N$ expansion which picks out definite topologies for these surfaces. This result is an existence proof for a string formulation of QCD. However, the weights of the surfaces are extremely complicated 
and it is not at all clear how to take the continuum limit.

From quite general considerations we expect that the large $N$ limit of QCD is quite smooth, and should exhibit almost all of the qualitative features of theory. Thus an expansion in powers of $\frac{1}{3}$ or $\left(\frac{1}{3}\right)^{2}$ might be quite good. The longstanding hope has been to find an equivalent (dual) description of QCD as some kind of string theory, which would be useful in to calculate properties of the theory in the infrared.

The problems with this approach are many. First, if QCD is describable as a string theory it is not as simple a theory as that employed for critical strings. It appears to be easier to guess the string theory of everything than to guess the string theory of QCD. Most likely the weights of the surfaces that one would have to sum over will depend on the extrinsic geometry of the surface and not only its intrinsic geometry. We know very little about such string theories. Also there are reasons to believe that a string formulation would require many (perhaps an infinite) new degrees of freedom in addition to the coordinates of the string. Finally, there is the important conceptual problem-how do strings manage to look like particles at short distances. The one thing we know for sure about QCD is that at large momentum transfer hadronic scattering amplitudes have canonical powerlike behavior in the momenta, up to calculable logarithmic corrections. String scattering, on the other hand, is remarkably soft. Critical string scattering amplitudes have, for large momentum transfer, Gaussian fall-off [3]. How do QCD strings avoid this? ${ }^{\dagger}$

* There is also the problem that for large $N$ there is typically a phase transition between the strong and weak coupling regimes [2].

$\dagger$ Recently there have been some interesting speculations regarding this problem $[4]$. 


\section{Two Dimensional QCD}

Two dimensional QCD $\left(\mathrm{QCD}_{2}\right)$ is the perfect testing ground for the idea that gauge theories can be equivalent to string theory. First, many features of the theory are stringier in two than in four dimensions. For example, linear confinement is a perturbative feature which is exact at all distances. Most important is that the theory is exactly solvable. This is essentially because in two dimensions gluons have no physical, propagating degrees of freedom, there being no transverse dimensions. In fact $\mathrm{QCD}_{2}$ is the next best thing to a topological field theory. The correlation functions in this theory will depend, as we shall see, only on the topology of the manifold on which formulate the theory and on its area. For this reason we will be able to solve the theory very easily and explicitly.

Consider for example the expectation value of the Wilson loop for pure $\mathrm{QCD}_{2}, \operatorname{Tr}_{R} P e^{\oint_{C} A_{\mu} d x^{\mu}}$, for any contour, $C$, which does not intersect itself. Choose an axial gauge, say $A_{1}=0$, then the Lagrangian is quadratic, given by $\frac{1}{2} \operatorname{Tr} E^{2}$, where $E=\partial_{1} A_{0}$ is the electric field. The Wilson loop describes a pair of charged particles propagating in time. This source produces, in two dimensions, a constant electric field. The Wilson loop is then given by the exponential of the constant energy of the pair integrated over space and time. This yields,

$$
\operatorname{Tr}_{R} P e^{\oint_{C} A_{\mu} d x^{\mu}}=e^{-g^{2} C_{2}(R) A}
$$

where $g$ is the gauge coupling, $C_{2}(R)$ the quadratic Casimir operator for representation $R$ and $A$ the area enclosed by the loop. The expectation value of more complicated Wilson loops that do self intersect can also be calculated. Kazakov and Kostov worked out a set of rules for such loops in the large $\mathrm{N}$ limit [6]. They are quite complicated. $\mathrm{QCD}_{2}$ with quarks is also soluble, at least in the large $N$ limit. The meson spectrum was solved for $N \rightarrow \infty$ by 't Hooft. It consists of an infinite set of confined mesons with masses $m_{n}$ that increase as $m_{n}^{2} \sim n$. This provides one with a quite realistic and very instructive model of quark confinement [1], [7].

Is $\mathrm{QCD}_{2}$ describable as a string theory? The answer is not known, although there is much evidence that the answer is yes. I shall describe below a study that I have carried out to investigate this issue [8]. 
To simplify matters I shall discard the quarks and consider the pure gauge theory. This would correspond to a theory of closed strings only, quarks are attached to the ends of open strings. We shall consider the partition function for a $U(N)$ or $S U(N)$ gauge theory, on an arbitrary Euclidean manifold $\mathcal{M}$,

$$
\mathcal{Z}_{\mathcal{M}}=\int\left[\mathcal{D} A^{\mu}\right] e^{-\frac{1}{4 g^{2}} \int_{\mathcal{M}} d^{2} x \sqrt{g} \operatorname{Tr} F^{\mu \nu} F_{\mu \nu}}
$$

One might think that in the absence of quarks the theory is totally trivial, since in two dimensions there are no physical gluon degrees of freedom. This is almost true, however the free energy of the gluons will depend non-trivially on the manifold on which they live. In fact, one cannot simply gauge the gluons away. If, for example, $\mathcal{M}$ contains a non-contractible loop $C$, then if $\operatorname{Tr} P e^{\oint_{C} A_{\mu} d x^{\mu}} \neq 1$, one can not gauge $A_{\mu}$ to zero along $C$. Thus, the partition function will be sensitive to the topology of $\mathcal{M}$.

Although non-trivial the theory is extremely simple, almost as simple as a topological theory. It is easy to see that the partition function will only depend on the topology and on the area of the manifold $\mathcal{M}$. This is because the theory is invariant under all area preserving diffeomorphisms. To demonstrate this note that the two-dimensional field strength can be written as $F_{\mu \nu}=\epsilon_{\mu \nu} f$, where $\epsilon_{\mu \nu}$ is the anti-symmetric tensor and $f$ a scalar field. Thus the action is $S=\int \operatorname{Tr} f^{2} d \mu$, where $d \mu=\sqrt{g} d^{2} x$ is the volume form on the manifold. This action is independent of the metric, except insofar as it appears in the volume form. Therefore the theory is invariant under area preserving diffeomorphisms $\left(W_{\infty}\right)$. The partition function can thus only depend on the topology and on the area of the manifold $\mathcal{M}$,

$$
\mathcal{Z}_{\mathcal{M}}=\mathcal{Z}\left[G, g^{2}, A, N\right]=\mathcal{Z}\left[G, g^{2} A, N\right]
$$

where $G$ is the genus of $\mathcal{M}$.

Now we can state the conjecture that the logarithm of this partition function, the free energy, is identical to the partition function on some string theory, with target space $\mathcal{M}$, where we would identify the string coupling with $\frac{1}{N}$ and the string tension with $g^{2} N$, 


$$
\ln \left(\mathcal{Z}\left[G, g^{2} A, N\right]\right)=\mathcal{Z}_{\text {TargetSpace } \mathcal{M}}^{\text {String }}\left[g_{\text {st }}=\frac{1}{N}, \alpha^{\prime}=g^{2} N\right]
$$

As a candidate for the type of string theory I am thinking of consider the Nambu action, wherein

$$
\mathcal{Z}_{\mathcal{M}}^{\text {String }}=\sum_{h=\text { genus }}\left(g_{\mathrm{st}}\right)^{2 h-2} \int \mathcal{D} x^{\mu}(\xi) e^{\int d^{2} \xi \sqrt{g}}
$$

where $g$ is the determinant of the induced metric

$$
g=\operatorname{det}\left[g_{\alpha \beta}\right]=\operatorname{det}\left[\frac{\partial x^{\mu}}{\partial \xi_{\alpha}} \frac{\partial x^{\nu}}{\partial \xi_{\beta}} G_{\mu \nu}(x)\right]
$$

and $G_{\mu \nu}(x)$ is the metric on the manifold $\mathcal{M}$. This string theory, when the target space is two-dimensional, is indeed invariant under area preserving diffeomorphisms of the target space. To see this note that $\sqrt{g}=\left|\frac{\partial x^{\mu}}{\partial \xi^{\alpha}}\right| \sqrt{G}$, which is obviously unchanged by a map $x^{\mu} \rightarrow x^{\prime \mu}$ as long as $\left|\frac{\partial x^{\mu}}{\partial x^{\prime} \nu}\right|=1$. $^{\star}$ Unfortunately the only way we know to quantize this theory is to transform it into the Polyakov action, which upon quantization yields the standard non- critical string [10]. This is not what we want to do here, since the resulting theory is not even Lorentz invariant. Is there another quantization of the Nambu string that differs from the Polyakov quantization in two- dimensions? The answer is not known.

* Actually the Polyakov action with a two-dimensional target space also has a $W_{\infty}$ symmetry, although is is realized in a very nonlinear fashion. One might speculate that this is related to the well known $W_{\infty}$ symmetry of the $c=1$ string theory [9]. 


\subsection{Evaluation of the Partition Function}

The partition function for $\mathrm{QCD}_{2}$ can easily be evaluated by means of the following idea, originally due to Migdal [11]. The trick is to use a particular lattice regularization of the theory which is both exact and additive. For the lattice we take an arbitrary triangulation of the manifold and define the partition function as

$$
\mathcal{Z}_{\mathcal{M}}=\int \prod_{L} d U_{L} \prod_{\text {plaq. }} Z_{P}\left[U_{P}\right]
$$

where $U_{P}=\prod_{L \in \text { plaq. }} U_{L}$, and $Z_{P}\left[U_{P}\right]$ is some appropriate lattice action. Any action will do as long as it reduces in the continuum limit to the usual continuum action. Instead of the Wilson action, $Z_{P}(U)=$ $e^{-\frac{1}{g^{2}} \operatorname{Tr}\left(U+U^{\dagger}\right)}$, we shall choose the heat kernel action,

$$
Z_{P}=\sum_{R} d_{R} \chi_{R}\left(U_{P}\right) e^{-g^{2} C_{2}(R) A_{P}}
$$

where the sum runs over representations $R$ of $S U(N)$ (or $U(N)), d_{R}$ is the dimension of $R, \chi_{R}\left(U_{P}\right)$ the character of $U_{P}$ in this representation, $C_{2}(R)$ the quadratic Casimir operator of $R$ and $A_{P}$ the area of the plaquette.

It is easy to see, using the completeness of the characters to expand about $Z_{P} \stackrel{U_{L} \rightarrow 1+i A_{\mu} d x^{\mu}}{\rightarrow} \sum_{R} d_{R} \chi_{R}\left(U_{P}\right)=\delta\left(U_{P}-1\right)+\ldots$, that in the continuum limit of this theory reduces to ordinary Yang-Mills theory. What is special about the heat kernel action is that it is additive. Namely, we can integrate over each link on the triangulation, say $U_{1}$, which appears in precisely two triangles, using the orthogonality of the characters, $\int d V \chi_{a}(X V) \chi_{b}\left(V^{\dagger} Y\right)=\frac{\delta_{a b}}{d_{a}} \chi_{a}(X Y)$, and obtain,

$$
\int d U_{1} Z_{P_{1}}\left(U_{2} U_{3} U_{1}\right) Z_{P_{2}}\left(U_{1}^{\dagger} U_{4} U_{5}\right)=Z_{P_{1}+P_{2}}\left(U_{2} U_{3} U_{4} U_{5}\right)
$$

This formula expresses the unitarity of the action, since in fact $Z_{P}(U)=$ $\left\langle U\left|e^{-g^{2} A \Delta}\right| 1\right\rangle$, where $\Delta$ is the Laplacian on the group. 
We can use this remarkable property of the heat kernel action to argue that the lattice representation is exact and independent of the triangulation. This is because we can use (3.9) in reverse to add as many triangles as desired, thus going to the continuum limit. On the other hand we can use (3.9) to reduce the number of triangles to the bare minimum necessary to capture the topology of $\mathcal{M}_{G}$. A two- dimensional manifold of genus $G$ can be described by a $4 G$-gon with identified sides: $a_{1} b_{1} a_{1}^{-1} b_{1}^{-1} \ldots a_{G} b_{G} a_{G}^{-1} b_{G}^{-1}$. The partition function can be written using this triangulation as,

$\mathcal{Z}_{\mathcal{M}_{G}}=\sum_{R} d_{R} e^{-g^{2} C_{2}(R) A} \int \prod \mathcal{D} U_{i} \mathcal{D} V_{i} \chi_{R}\left[U_{1} V_{1} U_{1}^{\dagger} V_{1}^{\dagger} \ldots U_{G} V_{G} U_{G}^{\dagger} V_{G}^{\dagger}\right]$

We can now evaluate the partition function using the orthogonality of the characters and the relation, $\int \mathcal{D} U \chi_{a}\left[A U B U^{\dagger}\right]=\frac{1}{d_{a}} \chi_{a}[A] \chi_{a}[B]$, to obtain [12], [13],

$$
\mathcal{Z}_{\mathcal{M}_{G}}=\sum_{R} d_{R}^{2-2 G} e^{-\frac{\lambda A}{N} C_{2}(R)}
$$

where $\lambda \equiv g^{2} N$ is kept fixed. Thus we have an explicit expression for the partition function. It depends, as expected, only on the genus and the area of the manifold.

\subsection{The Large $\mathbf{N}$ expansion}

The formula (3.11) for the partition function is quite complicated, being written as a sum over all representations of $S U(N)$. The representations of $S U(N)$ or $U(N)$ are labeled by the Young diagrams, with $m$ boxes of length $n_{1} \geq n_{2} \geq n_{3} \geq \ldots n_{m} \geq 0$. Such a representation has, 


$$
\begin{aligned}
C_{2}(R) & =N \sum_{i=1}^{m} n_{i}+\sum_{i=1}^{m}\left(n_{i}+1-2 i\right) \\
d_{R} & =\frac{\Delta(h)}{\Delta\left(h^{0}\right)}, \quad h_{i}=N+n_{i}-1, \quad h_{i}^{0}=N-i \\
\Delta(h) & =\prod_{1 \leq i<j \leq N}\left(h_{i}-h_{j}\right) .
\end{aligned}
$$

Thus we have a very explicit sum and one can, in principle, expand each term in powers of $\frac{1}{N}$ and evaluate the sum.

What do we expect if the string conjecture is correct? Consider the expansion in powers of $\frac{1}{N}$ of the free energy,

$$
\ln \left[\mathcal{Z}_{\mathcal{M}_{G}}\right]=\sum_{g=0}^{\infty} \frac{1}{N^{2 g-2}} f_{g}^{G}(\lambda A)
$$

If this were given by a sum over maps of a two-dimensional surface of genus $g$ onto a two-dimensional surface of dimension $G$ we would expect that $f_{g}^{G}(\lambda A) \sim\left(\frac{1}{N}\right)^{2 g-2} e^{-\lambda A n}$, where $n$ is the winding number of the map, i.e. the topological index that tells us how many times the map $x(\xi)$ covers $\mathcal{M}$. This is the integral of the Jacobian of the map $\xi \rightarrow x$, $\int d^{2} \xi \operatorname{det}\left[\frac{\partial x^{\mu}}{\partial \xi^{i}}\right]$, which differs from the Nambu area, $\int d^{2} \xi\left|\operatorname{det}\left[\frac{\partial x^{\mu}}{\partial \xi^{i}}\right]\right|$, since the surface can fold over itself.

Now there is a minimum value that $G$ can take, given the genus $G$ of the target space and the winding number $n$. Thus for example there are no smooth maps of a sphere onto a torus or a torus onto a genus two surface. Similarly there are no smooth maps of a genus $g$ surface onto a genus $g$ surface that wind around it more than once. To get an idea of the bound consider holomorphic maps, in which case the RiemannHurwitz theorem state states that $2(g-1)=2 n(G-1)+B$, where $B$ is the total branching number. In the case of smooth maps there seems to be the following bound [14],

$$
2(g-1) \geq 2 n(G-1)
$$

Thus if $\mathrm{QCD}_{2}$ is described by a string theory we would expect that 


$$
f_{g}^{G}(\lambda A)=\sum_{n}\left\{\begin{array}{ll}
0 & \text { if }(g-1)<n(G-1) \\
e^{-n \lambda A} \omega_{g}^{n}(A) & \text { otherwise }
\end{array} .\right.
$$

We can use these inequalities as tests of whether our conjecture is correct. To do this we need to expand (3.11) in powers of $\frac{1}{N}$.

The hardest case is that of the sphere $(G=0)$, since the sum over representations blow up rapidly and it is not even evident that there exists a tamed large $N$ expansion. We can break up the sum in (3.11) into a sum over representations with $n$ boxes in the Young tableaux since, for large $N, C\left(R_{n}\right) \stackrel{N \rightarrow \infty}{\rightarrow} N \sum_{i} n_{i}=N n$. Thus,

$$
Z_{G=0}=\sum_{R} d_{R}^{2} e^{-\frac{\lambda A}{N} C_{2}(R) \stackrel{N \rightarrow \infty}{\rightarrow}} \sum_{n} \sum_{R_{n}} d_{R_{n}}^{2} e^{-n \lambda A}(1+\ldots)
$$

To evaluate this we need to evaluate the following sum, $\sum_{R_{n}} d_{R_{n}}^{2}$. This can be done using a method of discrete orthogonal polynomials [8], yielding,

$$
\sum_{R_{n}} d_{R_{n}}^{2}=\left(\begin{array}{c}
N^{2}+n-1 \\
n
\end{array}\right)
$$

Then it follows that,

$$
Z_{G=0} \rightarrow \exp \left[-N^{2}\left[\ln \left(1-e^{-\lambda A}\right)+\frac{2 \lambda A e^{-2 \lambda A}}{\left(1-e^{-\lambda A}\right)^{2}}+\ldots\right]+O\left(N^{0}\right)+\ldots\right]
$$

Here there are no constraints implied by the inequality (3.15), but the structure of the expansion is very interesting.

The case of the torus, $(G=1)$, is some what simpler. One can easily derive that (for $S U(N)$ ) [8], 


$$
\begin{aligned}
Z_{G=1} & =\sum_{R} e^{-\frac{\lambda A}{N} C_{2}(R)} \rightarrow \exp \left[-N^{0} \ln \eta\left(-e^{-\lambda A}\right)+\right. \\
& \left.\frac{\lambda A}{N^{2}} \sum_{n=1}^{\infty} e^{-n \lambda A}\left[\sum_{a b=n} a^{2} b+\sum_{a b+c d=n} a c\right]+\ldots\right],
\end{aligned}
$$

where $\eta(x)=\prod_{n=1}^{\infty}\left(1-x^{n}\right)^{-1}$. This is totally consistent with the bound $g \geq 1$.

Most interesting is the case of $G>1$, where the inequalities are quite stringent. In this case one can easily derive [8],

$$
Z_{G} \rightarrow \sum_{n}\left(\frac{1}{N}\right)^{2 n(G-1)} e^{-n \lambda A} \sum_{r=\text { rep of } S_{n}}\left[\frac{n !}{d_{r}}\right]^{2(G-1)}
$$

where the sum is over representations of the symmetric group $S_{n}$ and $d_{r}$ is the dimension of the $\mathrm{r}^{\text {th }}$ representation of $S_{n}$. Not only is this in total accord with our expectations, but one can also show that $\omega_{g}^{n}(A)=$, for $g=1+n(G-1)$, is precisely the number of topologically inequivalent maps on the genus $g$ manifold onto the genus $G$ manifold with winding number $n[15]$.

So the large $\mathrm{N}$ expansion of $\mathrm{QCD}_{2}$ looks precisely like what we would expect from string considerations. What remains to be understood are the all the rational numbers that appear as coefficients of the powers of $e^{-\lambda A}$ and of $\frac{1}{N}$ in terms of the counting of maps of $\mathcal{M}_{g}$ onto $\mathcal{M}_{G}$. Some of these are understood, but not all. Then it remains to construct a string action that reproduces these counting rules. 


\section{Induced QCD}

\subsection{The Large $\mathbf{N}$ Limit of QCD}

QCD is hard to solve since it is a theory with no free, adjustable or small parameters. In pure QCD (no quarks) the only parameter we can adjust is the number of colors, $N$. Luckily, in the large $N$ limit QCD simplifies enormously, and this limit remains the best hope to yield an exact or controllable treatment of the theory. We know that as $N=\infty$ only planar graphs survive. More generally we know that in terms of the appropriate variables the large $N$ limit of gauge invariant observables is given, for $N=\infty$ by the master field, namely a solution of an appropriate classical equation of motion [16]. The large $N$ limit is in the nature of a semi-classical expansion, with $N$ playing the role of Planck's constant. Unlike the running coupling $N$ does not vary with momentum and we expect the large $N$ limit to be qualitatively correct for all momenta, to correctly capture the small distance asymptotic freedom of the theory as well as exhibit confinement at large distances. In the $N=\infty$ we should have an infinite spectrum of stable mesons and glueballs. Even baryons, bound states of $N$ quarks, are describable, in this limit, as solitons of the effective Lagrangian for the master field [17]. Thus the hope has survived that we could find an exact solution of QCD for $N=\infty$, which would yield the hadronic spectrum, and would be the starting point for a systematic large $N$ expansion which could allow us to calculate scattering amplitudes.

The standard method of solving a theory in the large $N$ limit is to find an appropriate saddlepoint for the partition function. In the case of QCD this is difficult. Consider the standard (Wilson) lattice formulation of the theory,

$$
\mathcal{Z}_{\mathrm{QCD}}=\int \prod_{L} \mathcal{D} U_{L} e^{-\sum_{\text {plaq. }} \frac{N}{g^{2}(a)} \operatorname{Tr}\left[\prod_{L} U_{L}+\text { h.c. }\right]}
$$

The integrand behaves as the exponential of an action that is of order $N^{2}$, thus one might hope to evaluate it by saddlepoint techniques. However, the measure is also of order $c^{N^{2}}$ and therefore one must somehow get rid of $N^{2}$ degrees of integration before this can be done. The reason QCD is not yet solved in the large $N$ limit is that no one knows how to reduce the theory to $N$ variables per site. 
Another theory which is also insoluble in the large $N$ limit is the non-critical string with $c>1$. Following the recent success of the matrix model solutions of string theory [18], we can construct such strings if we could deal with the large $N$ limit of a scalar matrix model in $D$ dimensions, say

$$
\mathcal{Z}_{D}^{\text {string }}=\int \prod_{i} \mathcal{D} \phi_{i} e^{-N \sum_{i} \operatorname{Tr} U\left(\phi_{i}\right)+N \sum_{i, \mu=1 \ldots D} \operatorname{Tr}\left(\phi_{i} \phi_{i+\mu}\right)},
$$

which describes a scalar field on a $D$ - dimensional lattice. The connection with string theory is made in the usual way, the Feynman diagrams of the perturbative expansion of (4.2) correspond, in an expansion in powers of $\frac{1}{N}$, to triangulations of two-dimensional surfaces. The scalar fields correspond to matter on this surface and thus, (4.2), could yield, at the appropriate critical point where the mean number of triangles diverges, a $c=D$ string theory. The standard approach to the large $N$-limit of such a theory is to diagonalize the matrices $\phi$, i.e. to pass to radial coordinates, $\phi_{i}=\Omega_{i} \lambda_{i} \Omega_{i}^{\dagger}$, where $\lambda_{i}$ is diagonal. In terms of these variables,

$$
\mathcal{Z}_{D}^{\text {string }}=\int \prod_{i} \mathcal{D} \lambda_{i} \mathcal{D} \Omega_{i} \Delta^{2}\left(\lambda_{i}\right) e^{-N \sum_{i} \operatorname{Tr} U\left(\lambda_{i}\right)+N \sum_{<i j>} \operatorname{Tr}\left(\lambda_{i} V_{i j} \lambda_{j} V_{i j}^{\dagger}\right)},
$$

where $V_{i j}=\Omega_{i} \Omega_{j}^{\dagger}$ and $\Delta(\phi)=\prod_{i<j}\left(\phi_{i}-\phi_{j}\right)$.

The next step is to integrate out the diagonalization matrices, $\Omega_{i}$. We can change variables from the $\Omega_{i}^{\prime} s$, defined on the sites to the $V_{i j}$ 's, defined on the links $\prod_{i} \mathcal{D} \Omega_{i}=\prod_{<i j>} \mathcal{D} V_{i j} \prod_{\text {plaq. }} \delta\left(1-\prod_{L} V_{i j}\right)$. The constraints arise since the $V_{i j}$ 's are pure gauge fields. If not for the constraints we could perform the integral over the $V_{i j}$ 's and reduce the integral to one over $N$ variables per site that could be evaluated by saddlepoint techniques. It is these constraints that have prevented the construction of strings with $c>1$.

Now let us combine these two models to consider QCD with adjoint scalar matter, 


$$
\begin{aligned}
& \mathcal{Z}_{\mathrm{QCD}}^{\mathrm{adj}}=\int \prod_{L} \mathcal{D} U_{L} \prod_{i} \mathcal{D} \phi_{i} e^{-N \sum_{i} \operatorname{Tr} U\left(\phi_{i}\right)+N \sum_{i, \mu=1 \ldots D} \operatorname{Tr}\left(\phi_{i} U_{\mu} \phi_{i+\mu} U_{\mu}^{\dagger}\right)} \\
& e^{-\frac{N}{g^{2}(a)} \sum_{\text {plaq. }}\left[\operatorname{Tr}\left(\prod_{L} U_{L}+\text { h.c. }\right)\right]} .
\end{aligned}
$$

This theory is invariant under standard gauge transformations, $\phi_{i} \rightarrow$ $V_{i} \phi_{i} V_{i}^{\dagger} ; \quad U_{\mu} \rightarrow V_{i} U_{\mu} V_{i+\mu}^{\dagger}$, which allow us to diagonalize the $\phi$ 's. However the presence of the Wilson action prevents us from handling this theory for large $N$. If set the gauge coupling to zero, we recover the previous model, since in this limit we can drop the Wilson action term, as long as we enforce the constraints, $\operatorname{tr}\left[U_{P}\right]=1$. However if we take the opposite limit, i.e. set $g=\infty$, then we can simply drop the Wilson action and the model will be soluble in the large $N$ limit. This is induced $Q C D$ [19].

Induced QCD has the one great advantage of being soluble, or at least reducible to a well defined master field equation. This is because the integral over the link matrices can now be performed. This is the famous Itzykson- Zuber integral [20],

$$
I(\phi, \chi) \equiv \int \mathcal{D} U e^{N \operatorname{Tr}\left[\phi U \chi U^{\dagger}\right]}=\frac{\operatorname{det}\left[e^{N \phi_{i} \chi_{j}}\right]}{\Delta(\phi) \Delta(\chi)}
$$

This formula is very profound, underlies all the analysis of the $c=1$, matrix model, and can be derived in many ways. One is the demonstration that the integral is given exactly by the WKB approximation, and the answer is simply the sum over the $N$ ! saddlepoints, for which are the $U$ are permutation matrices.

Although soluble this model appears to be very far from QCD, since asymptotic freedom instructs us to set the lattice coupling to zero, not infinity, in the continuum limit. However, Kazakov and Migdal argued that even though there is no kinetic term for the gauge field, it could be induced at large distances [19]. They argued that if one integrates out the scalar mesons (even in the case of noninteracting scalars with $U(\Phi)=\frac{1}{2} m^{2} \Phi^{2}$ ), then at distances large compared to $a$, one would induce in four dimensions an effective gauge interaction, 


$$
S_{\text {eff }}(U) \sim \frac{N}{96 \pi^{2}} \ln \left(\frac{1}{m^{2} a^{2}}\right) \operatorname{Tr} F_{\mu \nu}^{2}+\text { finite as } a \rightarrow 0 .
$$

This is simply the one loop vacuum graph for the scalars in a background gauge field, which is logarithmically divergent in four dimension. Now this looks very much like the ordinary Yang-Mills action, $\frac{1}{g^{2}(a)} \operatorname{tr} F_{\mu \nu} F^{\mu \nu}$, if we recall that asymptotic freedom tell us that $\frac{1}{g^{2}(a)}=\frac{11 N}{48 \pi^{2}} \ln \left(\frac{1}{M_{g}^{2} a^{2}}\right)$, where $M_{g}$ is a mass scale for QCD, say the glueball mass. We can therefore identify these two (the fact that there are $N^{2}$ scalars is crucial, as is the sign of the effective action which is due to the non-asymptotic freedom of the scalars.) If we do so then we find that, $M_{g}^{2}=m^{\frac{23}{11}} a^{\frac{1}{22}}$. Thus in the continuum limit the adjoint scalars become infinitely massive and decouple, but not before they have drive $\frac{1}{g^{2}}$ up, from zero at distance $a$ to the large QCD value atdistance $\frac{1}{m}$, where $\frac{1}{M_{g}}>>\frac{1}{m}>>a$. The basic idea is that the infrared slavery of the scalars, at the size of the lattice spacing, produces an effective gauge theory at a larger scale (much larger than the inverse scalar mass), which then produces the usual asymptotically free fixed point theory.

There are many problems with this idea. For one the hard gluons are not absent and their contribution will overwhelm that of the scalars at short distances. Their asymptotic freedom is more powerful than the infrared slavery of scalars. Another issue is that the above theory possesses a much larger symmetry than the $S U(N)$ gauge symmetry of the usual lattice action. It is not difficult to see that, in $\mathrm{D}$ dimensions, it is invariant under $(D-1) \times(N-1)$ extra local $U(1)$-gauge symmetries. This is because the transformation $U_{\mu}(x) \rightarrow V_{\mu}^{\dagger}(x) U_{\mu}(x) V_{\mu}(x+\mu a)$, leaves the action invariant as long as $V_{\mu}(x)$ is a unitary matrix that commutes with $\Phi(x)$. If $V_{\mu}(x)$ were independent of $\mu$ then this would be the ordinary gauge invariance. Thus we have $D-1$ new gauge symmetries, which are of course isomorphic to the special unitary transformations that commute with $\Phi[21]$. Thus $V_{\mu}(x)=D_{\mu}(x) \Omega(x)$, where $\Omega(x)$ is the unitary matrix that diagonalizes $\Phi$ and $D_{\mu}(x)$ is diagonal.

A subset of this symmetry is the, field independent, local $Z_{N}$ symmetry, $U_{\mu}(x) \rightarrow Z_{\mu} U_{\mu}(x) Z_{\mu}^{\dagger}$, where $Z_{\mu}$ is an element of the center of the group. This symmetry alone prevents the Wilson loop from acquiring an expectation value. A Wilson loop contains different links, and thus 
$W(C)=\left\langle\prod_{L \in C} U_{L}\right\rangle \rightarrow\left(\prod_{L} Z_{\mu}\right) W(C) \Rightarrow W(C)=0$. This symmetry must be broken if we are to recover the QCD fixed point from this formulation [22].

Finally, as we shall see, the simple Gaussian model is soluble and the answer is very simple and not equivalent to QCD [21]. However, there are interesting attempts to save the model and furthermore even if it does not yield a solution of QCD it might provide some interesting soluble matrix models which could yield new solutions of new string theories. Induced QCD is a matrix model and thus it corresponds to some kind of sum over surfaces. If we look at the Itzykson-Zuber integral we note that it could be expressed as,

$$
I(\phi, \chi)=\exp \left[\frac{1}{2} \operatorname{Tr} \phi^{2} \operatorname{Tr} \chi^{2}+a \operatorname{Tr} \phi^{4} \operatorname{Tr} \chi^{4}+\frac{b}{N^{2}}\left(\operatorname{Tr} \phi^{2}\right)^{2}\left(\operatorname{Tr} \chi^{2}\right)^{2}+\ldots\right] .
$$

These terms will affect the structure of the large $N$ expansion of the Feynman diagrams, and can be interpreted as yielding extra weights when the two-dimensional surfaces intersect [23]. Thus this model corresponds, perhaps, to some kind of string theory with weights that depend on the extrinsic geometry.

\subsection{Solution of the Gaussian model}

To try to solve the model of induced QCD we first integrate out he $U_{L}$ 's, then look for extrema of the effective action,

$S\left[\phi_{i}\right]=N^{2}\left[\frac{1}{N} \operatorname{Tr} \sum_{i} U\left(\phi_{i}\right)+\frac{1}{N^{2}} \sum_{i, \mu} \ln I\left(\phi_{i}, \phi_{i+\mu}\right)+\frac{1}{N^{2}} \sum_{i} \ln \Delta^{2}\left(\phi_{i}\right)\right]$.

In the large $\mathrm{N}$ limit the integral will be dominated by a translationally invariant saddlepoint for the density of eigenvalues of the matrices $\Phi_{i}, \rho(x) \equiv \frac{1}{N} \sum_{a=1}^{N} \delta\left(x-\phi_{a}\right)$. Migdal has derived the master field equation for the saddlepoint, using the Schwinger-Dyson equations that are satisfied by $I(\phi, \chi)[24]$. These are consequences of the fact that $I$ satisfies $\operatorname{tr}\left[\left(\frac{1}{N} \frac{\partial}{\partial \phi}\right)^{k}\right] I=\operatorname{tr}(\chi)^{k} I$. The net result is that one derives an equation for the function $F(z) \equiv \int d z \frac{\rho(\nu)}{z-\nu}$, whose imaginary part is $\operatorname{Im} F(\nu)=-\pi \rho(\nu)$, 


$$
\operatorname{Re} F(\lambda)=P \int \frac{d \nu}{2 \pi i} \ln \left[\frac{\lambda-\frac{1}{2 D} U^{\prime}(\nu)-\frac{D-1}{D} \operatorname{Re} F(\nu)+i \pi \rho(\nu)}{\lambda-\frac{1}{2 D} U^{\prime}(\nu)-\frac{D-1}{D} \operatorname{Re} F(\nu)-i \pi \rho(\nu)}\right] .
$$

This equation is much more complicated than the usual Riemann-Hilbert problem that one obtains for simple matrix models. It is sufficiently non-linear and complex that one might imagine that it describes QCD.

The master field equation simplifies dramatically for $D=1$. This is because in one dimension the gauge field can be gauged away completely, thus the model is equivalent to a scalar field on a one-dimensional lattice. The large $N$ limit of this model describes the $c=1$ string on a discrete target space, a model which has been solved in the double- scaling limit for small lattice spacing [25]. It undergoes a phase transition at a finite lattice spacing and it might be very instructive to use (4.9) to explore this phenomenon. sian,

In particular for the quadratic potential the path integral is Gaus-

$$
Z=\int \prod_{n} \mathcal{D} \Phi_{n} e^{-N \sum_{n} \operatorname{Tr}\left\{\frac{m^{2}}{2} \Phi_{n}^{2}-\Phi_{n} \Phi_{n+1}\right\}}
$$

Thus the eigenvalues of $\Phi$ will be given by the semi-circular distribution, namely $\pi \rho(\nu)=\sqrt{\mu-\frac{\mu^{2} \nu^{2}}{4}}$, where $\mu$ is determined by the mean of the squares of the eigenvalues, $\left\langle\frac{1}{N} \operatorname{Tr}\left(\Phi^{2}\right)\right\rangle=\frac{1}{\mu}$. It is therefore sufficient to calculate the expectation value of $\frac{1}{N} \operatorname{Tr}\left(\Phi^{2}\right)$, which is given by the one loop integral,

$$
\frac{1}{N} \operatorname{Tr}\left(\Phi^{2}\right)=\int_{-\pi}^{\pi} \frac{d p}{2 \pi} \frac{1}{m^{2}+2 \cos p}=\frac{1}{\sqrt{m^{4}-4}} \equiv \frac{1}{\mu} .
$$

It is easy to verify that this solves (4.9), using the fact that

$$
F(z)=\frac{\mu z}{2}-\sqrt{\frac{\mu^{2} z^{2}}{4}-\mu} ; \quad \operatorname{Re} F(\nu)=\frac{1}{2} \mu \nu
$$

However, if we return to (3.8), we see that the integral involved is of the same form for any $D$, as long as $\Re V^{\prime}(\nu)$ is linear in $\nu$. This suggests 
that we can find a solution of (4.9) with a semi-circular distribution of eigenvalues for a quadratic potential in any dimension [21].

Indeed, one can see that a semi-circular distribution of eigenvalues satisfies (4.9) for any $D$ as long as,

$$
\mu_{ \pm}(D)=\frac{m^{2}(D-1) \pm D \sqrt{m^{4}-4(2 D-1)}}{2 D-1} .
$$

This solution is much too trivial to describe QCD. In particular, for $D>1$ there is no sensible continuum limit of the model.

Is the solution unique? To see that it is note that in the master field equation the dimension of space-time enters only via the number of nearest neighbors of a given site, the coordination number of the lattice. The translationally invariant background scalar field is the same for any lattice with the same coordination number. The observables, say the scalar propagator, will of course depend on the full structure of the lattice, but not he background field. Therefore we can choose another simpler lattice with the same coordination number, say a Bethe lattice, which contains no closed loops. For such a lattice, as in the case of the $D=1$ model, the gauge field can be eliminated completely, and the model is equivalent to,

$$
Z_{\text {BetheLattice }}=\int \mathcal{D} \phi_{i} e^{-N \sum_{i} \operatorname{Tr} \frac{m^{2}}{2} \phi_{i}^{2}+N \sum_{<i j>} \operatorname{Tr}\left[\phi_{i} \phi_{j}\right]} .
$$

This model is easily soluble. We define $Z(\phi)$ to be the partition function of a branch of the Bethe lattice with coordination number $2 D$, so that $Z=\int \mathcal{D} \phi Z(\phi)^{2 D} e^{-\frac{m^{2}}{2} N \operatorname{Tr} \phi^{2}} . Z(\phi)$ satisfies the equation,

$$
Z(\phi)=\int \mathcal{D} \phi^{\prime} Z\left(\phi^{\prime}\right)^{2 D-1} e^{-\frac{m^{2}}{2} N \operatorname{Tr} \phi^{\prime 2}+N \operatorname{Tr}\left[\phi \phi^{\prime}\right]} .
$$

These equations are easily soluble. Take $Z(\phi)$ to have the form $Z(\phi)=$ $c e^{-N \frac{\alpha}{2} \operatorname{Tr} \phi^{2}}$, then (4.15) determines $\alpha$ to equal $\alpha=\frac{-m^{2} \pm \sqrt{m^{4}-4(2 D-1)}}{2(2 D-1)}$. Then (4.14) can be used to determine $\frac{1}{N} \operatorname{Tr} \phi^{2}=\frac{1}{m^{2}+2 \alpha D}$, which agrees precisely with $\frac{1}{\mu}$ as given by (4.13).

* I thank C. Bachas for emphasizing this point to me. 


\subsection{Prospects}

The simplest Gaussian model fails, but all hope is not lost. It is certainly possible to induce QCD if one introduces enough flavors of matter. The problem is that one then loses solubility. It might be that the self interactions of the scalars could be adjusted to drive the theory towards the asymptotically free fixed point. This hope has been pursued with great vigor by Migdal, who has also considered adding fermions, not too many so that the model remains soluble, so as to break the $Z_{N}$ symmetry [26]. Time will tell whether this will succeed. Even if it does not these model might yield a new class of interesting soluble matrix models which could teach us something about new classes of strings, perhaps strings that depend on extrinsic geometry. For this reason alone it is worth studying these models.

\section{REFERENCES}

1. G. 't Hooft, Nucl. Phys. B72, 461 (1974).

2. D. Gross and E. Witten, Phys. Rev. D21, 446(1980).

3. D. Gross and P. Mende,

4. J. Polchinski, Phys. Rev. Lett 68, 1267 (1992); M. Green, QMW91-24 (1991)

5. D. Weingarten, Phys. Lett. B90, 285 (1990); V. Kazakov and I. Kostov, Phys. Lett. B128, 316 (1983); I. Kostov Phys. Lett. B138, 191 (1984); Nucl. Phys. B179, 283 (1981); K. O'Brien and J. Zuber, Nucl. Phys. B253, 621 (1985)

6. V. Kazakov and I. Kostov, Nucl. Phys. B220, 167 (1983); I. Kostov Nucl. Phys. Lett. B265, 223 (1986).

7. C. Callan, N. Coote and D. Gross,

8. D. Gross, to be published

9. D. Gross and A. Polyakov, unpublished

10. A. Polyakov, Nucl. Phys. B40, 235 (1982).

11. A. Migdal, Zh. Eksp. Teor. Fiz. 69, 810 (1975)( Sov. Phys. Jetp. 42, 413). 
12. B. Rusakov, Mod. Phys. Lett. A5, 693 (1990).

13. E. Witten, Comm. Math. Phys,141, 153(1991; D.Fine, Comm. Math. Phys. 134, 273 (1990); M. Blau and G. Thompson, NIKHEF-H/91-09, MZ-TH/91-17.

14. S. Novikov and A. Schwarz, private communication.

15. D. Gross and W. Taylor, to be published

16. L. Yaffe, Rev. Mod. Phys.54, 407 (1982).

17. E. Witten, Nucl. Phys. B160, 57 (1979); V. Kazakov and A. Migdal,Phys. Lett. B103 , 214 (1981).

18. D. Gross and A.Migdal, Phys. Rev. Lett., 64, 717 (1990); M. Douglas and S. Shenker, Nucl. Phys. B335, 635 (1990); E. Brézin and V. Kazakov, Phys. Lett. 236B, 144 (1990).

19. V. Kazakov, and A. Migdal, PUPT-1322, May 1992.

20. Harish-Chandra, Amer. Jour. Math. 79, 87 (1957); C. Itzykson and J. Zuber, Jour. Math. Phys. 21, 411 (1980); J. Duistermaat and G. Heckman, Invent. Math. 69, 259 (1982).

21. D. Gross, PUPT 1335, (August 1992).

22. I. Kogan, G. Semenoff and N. Weiss, UBCTP 92-022, June 1992.

23. S. Das, A. Dhar, A. Sengupta and S. Wadia, Mod. Phys. Lett. A5, 1041 (1990); G. Korchemsky, UPRF-92-334 (1992).

24. A. Migdal, Princeton preprint PUPT-1323, June 1992.

25. D. Gross and I. Klebanov, Nucl. Phys. B334, 475 (1990).

26. A. Migdal, PUPT-1332, LPTENS-92/23 , PUPT-1343 ; S. Khoklachev and Yu. Makeenko, ITEP-YM-7-92 (August 1992); 